\title{
Atitudes de universitários da área da saúde relacionadas ao comportamento suicida
}

\author{
Attitudes of university students in the health field related to suicidal behavior \\ Actitudes de estudiantes universitarios en el campo de la salud relacionadas con la conducta suicida
}

Recebido: 08/04/2021 | Revisado: 16/04/2021 | Aceito: 20/04/2021 | Publicado: 05/05/2021

\author{
Maria Betânia Tinti de Andrade \\ ORCID: https://orcid.org/0000-0003-0329-1299 \\ Universidade Federal de Alfenas, Brasil \\ E-mail: betania.andrade@ unifal-mg.edu.br \\ Adriana Olimpia Barbosa Felipe \\ ORCID: https://orcid.org/0000-0002-4491-5750 \\ Universidade Federal de Alfenas, Brasil \\ E-mail: adriana.felipe@unifal-mg.edu.br \\ Ana Carolina Guidorizzi Zanetti \\ ORCID: https://orcid.org/0000-0003-0011-4510 \\ Universidade de São Paulo, Brasil \\ E-mail: carolzan@eerp.usp.br \\ Denismar Alves Nogueira \\ ORCID: https://orcid.org/0000-0003-2285-8764 \\ Universidade Federal de Alfenas, Brasil \\ E-mail: denismar.nogueira@unifal-mg.edu.br \\ Zélia Marilda Rodrigues Resck \\ ORCID: https://orcid.org/0000-0002-3752-8381 \\ Universidade Federal de Alfenas, Brasil \\ E-mail: zelia.resk@unifal-mg.edu.br \\ Carlos Alberto da Cruz Sequeira \\ ORCID: https://orcid.org/0000-0002-5620-3478 \\ Escola Superior de Enfermagem do Porto, Portugal \\ E-mail: carlossequeira@esenf.pt \\ Kelly Graziani Giacchero Vedana \\ ORCID: https://orcid.org/0000-0001-7363-2429 \\ Universidade de São Paulo, Brasil \\ E-mail: kellygiacchero@eerp.usp.br
}

\section{Resumo}

As atitudes relacionadas ao comportamento suicida entre universitários da saúde podem impactar busca por ajuda, bem como a assistência à pessoa com comportamento suicida. Assim, o presente estudo teve como objetivo analisar as atitudes relacionadas ao comportamento suicida entre graduandos de cursos da área de saúde e os fatores associados. Estudo transversal, quantitativo, realizado em 2018 com 747 estudantes universitários de uma instituição de ensino superior localizada no Sul de Minas Gerais. Para coleta dos dados foram autoaplicados um Questionário sociodemográfico, educacional e clínico; o Questionário de Atitudes frente ao Comportamento Suicida; a Escala de Esperança de Herth; a Avaliação do Risco de Suicídio da Minientrevista Neuropsiquiátrica; o Inventário de Depressão Maior e a Escala de Autoestima de Rosenberg. Os dados foram submetidos à análise estatística descritiva, teste de comparação de média, correlação e regressão múltipla. Os preditores de sentimentos negativos relacionados ao comportamento suicida foram: sexo feminino, não participar de atividades religiosas, menor percepção de competência profissional e atitudes moralistas. Foram preditores de competência profissional autopercebida: ter transtorno mental, ter mais esperança, presença risco de suicídio, não ter religião ou espiritualidade, menos sentimentos negativos e atitudes moralistas. Os preditores de maior compreensão do direito ao suicídio foram não participar de atividades religiosas, ter diagnóstico de transtorno mental, ler material específico sobre suicídio, ter menos sentimentos negativos, menos esperança e menos percepção da própria capacidade profissional. O estudo fornece conhecimento para subsidiar ações para promover formação profissional para qualificação da assistência a pessoas com comportamento suicida.

Palavras-chave: Atitude; Tentativa de suicídio; Suicídio; Estudantes de ciências da saúde; Enfermagem.

\begin{abstract}
Attitudes related to suicidal behavior among health university students can impact the search for help, as well as assistance to people with suicidal behavior. Thus, the present study aimed to analyze the attitudes related to suicidal behavior among undergraduate health students and the associated factors. Cross-sectional, quantitative study, carried out in 2018 with 747 university students from a higher education institution located in the south of Minas Gerais. For data collection, a sociodemographic, educational and clinical questionnaire was self-administered; the Attitude
\end{abstract}


Questionnaire towards Suicidal Behavior; Herth's Hope Scale; the Suicide Risk Assessment of the Neuropsychiatric Mini-interview; the Major Depression Inventory and the Rosenberg Self-Esteem Scale. The data were submitted to descriptive statistical analysis, mean comparison test, correlation and multiple regression. The predictors of negative feelings related to suicidal behavior were: female gender, not participating in religious activities, less perception of professional competence and moralistic attitudes. They were predictors of self-perceived professional competence: having a mental disorder, having more hope, having a risk of suicide, having no religion or spirituality, less negative feelings and moralistic attitudes. The predictors of greater understanding of the right to suicide were not participating in religious activities, being diagnosed with a mental disorder, reading specific material about suicide, having less negative feelings, less hope and less perception of one's professional ability. The study provides knowledge to support actions to promote professional training to qualify assistance to people with suicidal behavior.

Keywords: Attitude; Suicide attempted; Suicide; Students health occupations; Nursing.

\section{Resumen}

Las actitudes relacionadas con la conducta suicida entre los estudiantes universitarios de la salud pueden afectar la búsqueda de ayuda, así como la asistencia a las personas con conducta suicida. Así, el presente estudio tuvo como objetivo analizar las actitudes relacionadas con la conducta suicida entre estudiantes de pregrado de salud y los factores asociados. Estudio transversal, cuantitativo, realizado en 2018 con 747 estudiantes universitarios de una institución de educación superior ubicada en el sur de Minas Gerais. Para la recogida de datos se autoaplicado un cuestionario sociodemográfico, educativo y clínico; el Cuestionario de Actitudes hacia la Conducta Suicida; Escala de esperanza de Herth; la Evaluación del Riesgo de Suicidio de la Mini entrevista Neuropsiquiátrica; el Inventario de Depresión Mayor y la Escala de Autoestima de Rosenberg. Los datos fueron sometidos a análisis estadístico descriptivo, prueba de comparación de medias, correlación y regresión múltiple. Los predictores de sentimientos negativos relacionados con la conducta suicida fueron: género femenino, no participación en actividades religiosas, menor percepción de competencia profesional y actitudes moralistas. Fueron predictores de la competencia profesional autopercibida: tener un trastorno mental, tener más esperanza, tener riesgo de suicidio, no tener religión ni espiritualidad, menos sentimientos negativos y actitudes moralistas. Los predictores de una mayor comprensión del derecho al suicidio fueron no participar en actividades religiosas, ser diagnosticado con un trastorno mental, leer material específico sobre el suicidio, tener menos sentimientos negativos, menos esperanza y menos percepción de la propia capacidad profesional. El estudio aporta conocimientos para apoyar acciones de promoción de la formación profesional para capacitar la asistencia a personas con conducta suicida.

Palabras clave: Actitud; Intento de suicídio; Suicidio; Estudiantes del área de la salud; Enfermería.

\section{Introdução}

O comportamento suicida é um fenômeno complexo e multifatorial, resultante da interação de fatores de ordem filosófica, antropológica, psicológica, biológica e social (Zadravec \& Grad, 2013; Kim, Baek, Han, Lee, \& Yurgelun-Todd, 2015; Pereira, Willhelm, Koller, \& Almeida, 2018) e pode ser definido como todo e qualquer ato por meio do qual a pessoa causa lesão a si própria e inclui desde pensamentos de autodestruição, ameaças, gestos, tentativas de suicídio até o suicídio consumado (Werlang, Borges, \& Fensterseifer, 2005; Schlösser, Rosa, \& More, 2014).

Neste contexto é importante considerar que as atitudes de profissionais e universitários da área da saúde relacionadas ao comportamento suicida influenciam a qualidade da assistência a pessoas em risco de suicídio (Emergency Nurses Association, 2012; Saunders, Hawton, Fortune, \& Farrell, 2012; Carmona-Navarro, \& Pichardo-Martinez, 2012; Nebhinani, Nebhinani, Tamphasana, \& Gaikwad, 2013).

A atitude envolve aspectos cognitivos, afetivos e comportamentais, afeta a conduta em relação as pessoas, eventos ou objetivos. Assim, é uma forma de abordar e lidar com uma situação problema (Altmann, 2008).

A literatura referência que a capacitação dos profissionais de saúde sobre a temática tem contribuído para melhorar a assistência ao indivíduo em comportamento suicida (Nunes, Oliveira, \& Galvão, 2020).

A formação e o desenvolvimento de competências emocionais nos futuros profissionais de saúde são essenciais, uma vez que são estes que irão desempenhar as ações de reconhecimento dos fatores de risco, a prevenção e a assistência ao paciente com comportamento suicida (World Health Organization [WHO], 2000; Emergency Nurses Association, 2012; Carmona-Navarro, \& Pichardo-Martinez, 2012; Menon, 2013). 
Assim, é importante investigar como os universitários da área da saúde lidam com o comportamento suicida para planejar a formação de recursos humanos para melhoria da assistência. Portanto, são necessárias investigações que avaliem a compreensão das atitudes de profissionais e de estudantes universitários em relação às habilidades de manejo do suicídio, considerando-se variações existentes entre os diferentes países, culturas e épocas (Talseth \& Gilje, 2007; Nebhinani et al., 2013; Nunes et al., 2020) e incluindo variáveis ainda não exploradas em estudos prévios.

$\mathrm{Na}$ literatura, a associação entre atitudes relacionadas ao comportamento suicida e o sexo, a idade, o tempo de experiência clínica e a educação prévia, é variável e pouco esclarecida (Eskin, Voracek, Stieger, \& Altinyazar, 2011; Nebhinani et al., 2013; Kelly, Mccarthy, \& Sahm, 2014; Ramberg, Di Lucca, \& Hadlaczky, 2016; Flood et al., 2018, Öztürk \& Akin, 2018; Vedana, Santos, Zanetti, \& Miasso, 2020).

No contexto brasileiro ainda é incipiente os estudos que avaliam as atitudes frente ao comportamento suicida, sendo que alguns fatores como sexo, idade, período que cursa, leitura de material, cursos sobre a temática, experiência prévia, estiveram associados ou não as atitudes dos profissionais de saúde e universitários (Magalhães et al., 2014; Botti, Araújo, Costa, \& Almeida, 2015; Moraes, Magrini, Zanetti, Santos, \& Vedana, 2016; Giacchero Vedana et al., 2017; Storino et al., 2018; Kirchner \& Queluz, 2019; Nunes et al., 2020). Ressalta-se que todos estes estudos foram conduzidos com o QUACS (Botega et al., 2005), instrumento a ser utilizado no presente estudo.

$\mathrm{Na}$ busca de conhecer a formação, a conduta e as reações emocionais dos profissionais de saúde mental sobre o comportamento suicida, o estudo de Oliveira, Collares, Noal, e Dias (2016) apontou o déficit na formação destes, o que pode contribuir para o desconforto emocional e limitar as possibilidades de estratégias para lidar com os pacientes de risco.

Reisdorfer et al. (2015) investigaram o conhecimento e as estratégias de intervenções de profissionais de enfermagem relacionadas ao comportamento suicida e encontraram fragilidades no conhecimento acerca do tema e a necessidade destes profissionais de se qualificarem a fim de proporcionar melhoria no cuidado as pessoas que tentaram suicídio ou estejam em situações de risco.

Nessa perspectiva, é relevante ampliar o conhecimento e as atitudes dos universitários frente ao comportamento suicida e os fatores envolvidos neste contexto. O conhecimento produzido no estudo poderá subsidiar intervenções, investigações e programas de ensino para qualificar a formação de recursos humanos para a assistência à pessoa com comportamento suicida.

Este estudo teve como objetivo analisar as atitudes relacionadas ao comportamento suicida entre graduandos de cursos da área de saúde e os fatores associados.

\section{Metodologia}

Estudo transversal de abordagem quantitativa (Aragão, 2011). A pesquisa quantitativa aborda um grupo de procedimentos sistemáticos ordenados para coletar dados, partindo do estabelecimento do problema e da escolha dos conceitos para solucioná-los (Polit, \& Beck, 2011). Conduzido com graduandos da área da saúde que estavam matriculados a partir do $5^{\circ}$ período nos cursos de Biomedicina, Enfermagem, Farmácia, Fisioterapia, Nutrição, Medicina e Odontologia pertencentes a uma instituição pública de ensino superior, localizada no Sul de Minas Gerais. A escolha de graduandos nos últimos semestres foi fundamentada na importância de incluir estudantes com maior probabilidade de contato teórico ou prático com a temática. Foram excluídos graduandos menores de 18 anos, ausentes no momento da coleta de dados ou que preenchessem menos que $50 \%$ dos instrumentos. A população de graduandos dos cursos da área de saúde no período estudado totalizava 1090 acadêmicos, 340 foram excluídos, 339 estavam ausentes no momento da coleta, 01 respondeu menos que 50\% dos instrumentos e três (um acadêmico do Curso de Nutrição e dois do Curso de Medicina) recusaram participar da pesquisa. Assim, a amostra foi composta por 747 estudantes. A coleta de dados foi realizada entre maio e junho de 2018, após a 
aprovação do Comitê de Ética em Pesquisa da Instituição proponente (Parecer - $\mathrm{n}^{\circ}$ 2.392.345) e da Instituição coparticipante (Parecer $-n^{\circ}$ 2.398.238). Assim, os coordenadores de todos os cursos de graduação elegíveis foram contatados, apresentado os objetivos do estudo, solicitado autorização para a coleta de dados e a indicação de um professor de cada período do curso para facilitar o agendamento do dia e horário para a coleta. A coleta foi realizada no início ou no término das aulas de forma a não prejudicar o desenvolvimento das atividades. Após esclarecimento, os graduandos que aceitaram participar voluntariamente do estudo receberam o Termo de Consentimento Livre e Esclarecido e os instrumentos para coleta de dados. O tempo de preenchimento dos instrumentos foi de 20 a 35 minutos e todos os instrumentos foram autoaplicáveis.

Os instrumentos utilizados foram: roteiro semiestruturado validado para a coleta dos dados sociodemográficos, educacionais e clínicos, com as seguintes variáveis: idade, sexo (biológico), identificação de gênero, orientação sexual, curso, período do curso, participação em eventos, cursos e/ou palestras sobre prevenção de suicídio, participação em disciplina sobre psiquiatria e/ou saúde mental, diagnóstico de doença mental, tratamento com psicofármacos, acompanhamento em saúde mental, crença religiosa ou espiritualidade, apoio das pessoas com as quais convive, ter familiar ou amigo próximo com pensamento suicida ou tentativa de suicídio e familiar ou amigo próximo que morreu por suicídio.

O Questionário de Atitudes Frente ao Comportamento Suicida (QUACS) é o único instrumento validado no Brasil que avalia atitudes de profissionais em relação ao comportamento suicida, de fácil manuseio, abordagem individual e autoaplicável (Botega et al., 2005). Não apresenta pontos de corte dos escores que categorizem os resultados e pode ser analisado por meio dos três fatores, ou pela análise dos 21 itens isoladamente. No presente estudo optou-se em utilizar o agrupamento em três fatores que conseguem analisar as atitudes nos seus componentes afetivo, cognitivo e comportamental: 1Sentimentos negativos em relação ao paciente - quanto maior a pontuação nesse fator, maior a presença de sentimentos negativos; 2- Percepção de capacidade profissional - uma maior pontuação pode significar profissionais mais confiantes em lidar com indivíduos com comportamento suicida; e 3- Direito ao Suicídio - uma maior pontuação pode representar uma atitude menos "moralista/judiciosa". A pontuação de cada um dos três fatores pode variar entre zero e 30 pontos. Cada afirmação é seguida por uma escala visual analógica de dez centímetros (10 pontos) que variam desde "discordo totalmente", a “concordo totalmente" (Botega et al., 2005).

A Escala de Esperança de Herth (EEH), foi construído por Herth em 1992, traduzido e validado para o contexto brasileiro por Sartore e Grossi em 2008. É um instrumento que mensura o nível de esperança dos indivíduos, de fácil e rápida aplicação, composto por 12 itens escritos de forma afirmativa tipo Likert de 4 pontos, variando de 1 a 4 , sendo que 1 indica "discordo completamente" e o 4 indica "concordo completamente". O escore total varia de 12 a 48 pontos, sendo que, quanto maior o escore, mais alto o nível de esperança (Sartore \& Grossi, 2008).

Para avaliação do risco de suicídio, foi utilizado apenas o módulo C da Minientrevista Neuropsiquiátrica Internacional (M.I.N.I.), que é uma entrevista diagnóstica padronizada, de domínio público, simples e breve, a qual foi traduzida e adaptada por Amorim, 2000. Composta por perguntas que variam de pensamentos durante o último mês e ao longo da vida, com respostas dicotômicas (sim ou não). O escore do instrumento pode variar de um a 33 pontos, sendo classificado como risco de suicídio - 1 a 5 pontos (baixo), 6 a 9 pontos (moderado) e maior ou igual a 10 pontos (alto). A Escala de Risco de Suicídio foi recategorizada em sem risco (categoria proposta pelo instrumento, quando a pontuação foi igual a zero) e com risco (que reuniu as opções: baixo risco - 1 a 5 pontos; moderado - 6 a 9 pontos e alto - maior ou igual a 10 pontos) (Amorim, 2000).

O Inventário de Depressão Maior (IDM) permite avaliar a presença de transtorno depressivo, a severidade e a gravidade dos sintomas, nas duas últimas semanas, composto por questões afirmativas, a serem respondidas numa escala tipo Likert, com escores que podem variar de zero a cinco para cada item, no qual, zero - nenhuma vez, e o cinco - o tempo todo, varia de zero a 50 pontos, sendo que quanto maior o escore, maior a presença de transtorno depressivo e a magnitude dos 
sintomas depressivos. Neste estudo o instrumento foi codificado como: com sintomas depressivos (com escore maior ou igual a 16) e sem sintomas depressivos (com escore menor que 16 pontos) (Parcias et al., 2011).

Outro instrumento utilizado foi a Escala de Autoestima de Rosenberg (EAR) traduzida e validada para o contexto brasileiro por Hutz em 2000, tendo sua validação e normatização em 2011 por Hutz e Zanon. É uma escala que possui dez afirmações que avalia a autoestima global, desses dez itens, cinco avaliam sentimentos positivos do indivíduo sobre si mesmo e os outros cinco referem uma visão autodepreciativa, ou seja, avaliam sentimentos e autoimagem negativa. Os itens são escritos de forma afirmativa tipo Likert, de 1 a 4 pontos, variando entre - concordo totalmente a discordo totalmente. O escore total varia de 10 a 40 pontos, sendo que, quanto maior o escore, maior a autoestima (Hutz \& Zanon, 2011). A classificação proposta por Gomes \& Silva (2013) se refere a: satisfatória ou alta (maior que 31 pontos), média (entre 21 e 30 pontos) e insatisfatória ou baixa (menor que 20 pontos. Neste estudo, foi recategorizada em autoestima alta (maior ou igual a 31 pontos) e baixa (que reuniu as opções de resposta classificadas como média - entre 21 e 30 pontos e insatisfatória ou baixa - menor que 20 pontos).

Tabulou-se os dados em programa estatístico, para análise estatística descritiva, teste de comparação de média, correlação e regressão. Devido ao caráter assimétrico da distribuição das variáveis analisadas avaliada pelo Teste de Shapiro-Wilk, aplicou-se o Teste Mann Whitney e o Teste t, usado para o caso em que as variáveis apresentavam distribuição normal.

O coeficiente de correlação Spearman foi utilizado para avaliar a associação entre as atitudes relacionadas ao comportamento suicida, risco de suicídio, sintomas depressivos, esperança e autoestima. O coeficiente de correlação de Spearman assume os valores de -1 até +1 , sendo que, o valor positivo indica correlação direta e o negativo uma correlação inversa (Miot, 2018). Além disso, pode ser classificado como: fraco $(0<\mathrm{r}<0,4)$, moderado $(0,4<\mathrm{r}<0,7)$ e forte $(0,7<\mathrm{r}<$ 1,0) (Siqueira \& Tibúrcio, 2011).

A regressão múltipla foi realizada com o objetivo de avaliar o efeito das variáveis associadas ao desfecho atitudes relacionadas com comportamento suicida e as variáveis dependentes. Todas as variáveis foram inseridas no modelo, independentemente do resultado da análise bivariada, utilizou-se a deleção individual das variáveis até a obtenção do modelo final, com o uso do método stepwise.

Para todas as análises, considerou-se nível de significância de 5\%, ou seja, os dados foram estatisticamente significantes para $\mathrm{p}<0,05$.

\section{Resultados}

Participaram do estudo 747 estudantes de graduação da área de saúde, dos cursos de Biomedicina, Enfermagem, Farmácia, Fisioterapia, Nutrição, Medicina e Odontologia, a maioria dos graduandos era do sexo feminino, heterossexual, com idade menor que 25 anos, estarem entre o $5^{\circ}$ e o $7^{\circ}$ período, com crença religiosa, participavam de atividades religiosas e satisfeitos com o apoio que recebiam de familiares e amigos.

Os escores médios obtidos no QUACS sobre o direito ao suicídio $(15,4)$ foram mais elevados do que os obtidos na competência profissional autopercebida $(11,3)$ e nos sentimentos negativos $(10,5)$, o que representa maior concordância com afirmações menos moralistas do que com a competência profissional autopercebida ou com sentimentos negativos.

\section{Sentimentos negativos (Fator 1)}

No teste de comparação os escores de sentimentos negativos foram associados ao sexo feminino $(\mathrm{p}=<0,001)$, heterossexuais ( $\mathrm{p}=0,018)$, não participar eventos para prevenção $(\mathrm{p}=0,002)$ e não ler material sobre suicídio ( $\mathrm{p}=0,012)$, os quais estiveram associados a mais sentimentos negativos. Não foram encontradas associações entre os sentimentos negativos com a 
idade, a crença religiosa, a participação em atividades religiosas, a satisfação com apoio, o contato com familiar e pessoa próxima com comportamento suicida, o período do curso, a participação de disciplina sobre psiquiatria/saúde mental, o transtorno mental, o uso de psicofármacos, a terapia em saúde mental, o risco de suicídio, os sintomas depressivos e a autoestima.

O teste de Correlação Spearman verificou-se correlação negativa fraca entre o fator 1 (sentimentos negativos) e o fator 2 (competência profissional autopercebida) $(\mathrm{r}=-0,310 ; \mathrm{p}=<0,001)$ e o fator 3 (direito ao suicídio) $(\mathrm{r}=-0,090 ; \mathrm{p}=0,014)$, ou seja, os sentimentos negativos eram mais elevados quando a competência profissional e a compreensão do direito ao suicídio eram menores.

Todas as variáveis sociodemográficas, contato com pessoa próxima com comportamento suicida, educacionais e clínicos foram incluídas em modelos de regressão linear múltipla que tiveram como variável resposta as atitudes relacionadas ao comportamento suicida. As mulheres ( $\mathrm{p}=0,001)$ e aqueles que não participam de atividades religiosas $(\mathrm{p}=0,009)$ apresentavam mais sentimentos negativos em relação ao indivíduo com comportamento suicida. Os estudantes mais confiantes em lidar com indivíduos com comportamento suicida $(p<0,001)$ e os com atitudes menos moralistas $(p<0,001)$ com relação ao direito ao suicídio apresentaram menos sentimentos negativos (Tabela 1).

Tabela 1 - Análise dos fatores associados aos sentimentos negativos, segundo modelo de regressão linear múltipla, 2018.

\begin{tabular}{lcccc}
\hline \multicolumn{1}{c}{ Variável } & Parâmetro & $\mathbf{p}$ & \multicolumn{2}{c}{ IC 95\% } \\
\hline Sexo (feminino) & 1,588 & $\mathbf{, 0 0 1}$ &, 635 & 2,541 \\
Disciplina sobre psiquiatria/saúde mental (não) &,- 874 &, 070 & $-1,715$ &,- 034 \\
Participação em atividades religiosas (não) & 1,160 &, $\mathbf{0 0 9}$ &, 285 & 2,034 \\
F2 - Competência profissional autopercebida &,- 287 & $\mathbf{< , 0 0 1}$ &,- 358 &,- 216 \\
F3 - Direito ao suicídio &,- 133 & $<, 001$ &,- 193 &,- 074 \\
Constant & 14,335 & $<, 001$ & 12,777 & 15,892 \\
\hline
\end{tabular}

Modelo de regressão linear múltipla; $\mathrm{p}$ - valor de p; IC - intervalo de confiança. Fonte: Autores.

\section{Competência profissional autopercebida (Fator 2)}

Constatou-se maior competência profissional autopercebida o sexo masculino $(\mathrm{p}=0,001)$, ler material sobre suicídio ( $\mathrm{p}=0,014)$, participar de eventos sobre prevenção do suicídio $(\mathrm{p}=0,003)$, autoestima alta $(\mathrm{p}<0,001)$ e não ter sintomas depressivos $(\mathrm{p}=0,010)$. Não foram observadas associações entre a competência profissional autopercebida com a idade, a orientação sexual, a crença religiosa, a participação em atividades religiosas, a satisfação com apoio, o contato com familiar e pessoa próxima com comportamento suicida, o período do curso, a disciplina sobre psiquiatria/saúde mental, o transtorno mental, o uso de psicofármacos, a terapia em saúde mental e o risco de suicídio.

O fator 2 (competência profissional autopercebida) apresentou uma correlação negativa de fraca magnitude com o fator 3 (direito ao suicídio) ( $r=-0,081 ; p=0,027)$ e os sintomas depressivos ( $r=-0,110 ; p=0,003)$ e apresentou correlação positiva fraca com a esperança $(r=0,171 ; p=<0,001)$ e a autoestima $(r=0,146 ; p=<0,001)$. Assim, a confiança profissional para lidar com os indivíduos com comportamento suicida foi maior entre pessoas com mais esperança, maior autoestima, menos sintoma depressivos e menor compreensão do direito ao suicídio.

Na regressão linear múltipla, os estudantes sem religião ou espiritualidade $(\mathrm{p}=0,017)$, que não participavam de atividades religiosas, que referiram ter transtorno mental $(\mathrm{p}=0,001)$, tinham mais esperança $(\mathrm{p}<0,001)$ e apresentaram risco de suicídio $(\mathrm{p}=0,008)$ eram mais confiantes em lidar com os indivíduos com comportamento suicida. Os estudantes com mais sentimentos 
negativos $(\mathrm{p}<0,001)$ e com atitudes menos moralistas $(\mathrm{p}=0,001)$ se percebiam menos competentes para lidar com os indivíduos em comportamento suicida (Tabela 2).

Tabela 2 - Análise dos fatores associados às atitudes relacionadas à competência profissional autopercebida, segundo modelo de regressão linear múltipla, 2018.

\begin{tabular}{lcccc}
\hline \multicolumn{1}{c}{ Variável } & Parâmetro & $\mathbf{p}$ & \multicolumn{2}{c}{ IC 95\% } \\
\hline Religião (não) & 12,600 & $\mathbf{, 0 1 7}$ & 2,277 & 22,923 \\
Participação em atividades religiosas (não) & 0,978 & $\mathbf{, 0 3 4}$ &, 074 & 1,882 \\
Transtorno mental (sim) & 1,850 & $\mathbf{, 0 0 1}$ &, 792 & 2,908 \\
F1 - Sentimentos negativos &,- 328 & $<, 001$ &,- 406 &,- 251 \\
F3- Direito ao suicídio &,- 110 & $\mathbf{, 0 0 1}$ &,- 174 &,- 046 \\
Risco de suicídio (com risco) & 1,501 & $\mathbf{, 0 0 8}$ &, 396 & 2,606 \\
Esperança &, 255 & $\mathbf{< , 0 0 1}$ &, 174 &, 337 \\
Constant & 5,590 &, 003 & 1,974 & 9,205 \\
\hline
\end{tabular}

Modelo de regressão linear múltipla; p - valor de p; IC - intervalo de confiança. Fonte: Autores.

\section{Atitudes condenatórias (Fator 3)}

As atitudes relacionadas ao direito ao suicídio foram associadas ao sexo masculino ( $\mathrm{p}=0,001)$, não heterossexuais $(\mathrm{p}=<0,001)$, insatisfeitos com o apoio/suporte social $(\mathrm{p}=<0,001)$, não praticantes de crença religiosa ou espiritualidade $(\mathrm{p}=<0,001)$, não participar de atividades religiosas $(\mathrm{p}=<0,001)$, estar matriculado entre o $5^{\circ}$ e o $7^{\circ}$ período do curso $(\mathrm{p}=0,012)$, ler material específico sobre suicídio $(\mathrm{p}=0,001)$, com baixa autoestima $(\mathrm{p}<0,001)$, ter risco para o suicídio $(\mathrm{p}<0,001)$, com sintomas depressivos ( $<<0,001)$, que referiram ter transtorno mental $(p<0,001)$, usar psicofármacos $(p=0,043)$ e fazer terapia $(\mathrm{p}=0,003)$, se associaram a atitudes menos moralistas. Não foram estatisticamente significantes para o direito ao suicídio as variáveis idade, contato com familiar e pessoa próxima com comportamento suicida, disciplina sobre psiquiatria/saúde mental e eventos sobre prevenção de suicídio.

Evidenciou-se correlação negativa fraca entre F3 (direito ao suicídio) com a autoestima $(r=-0,235 ; \mathrm{p}=<0,001)$ e a esperança $(r=-0,307 ; p=<0,001)$ e correlação positiva fraca com os sintomas depressivos $(r=0,207 ; p=<0,001)$ e o risco de suicídio ( $\mathrm{r}=0,229 ; \mathrm{p}=<0,001)$. Assim, houve maior compreensão do direito ao suicídio entre as pessoas com menor autoestima, menos esperança, mais sintomas depressivos e maior risco de suicídio.

$\mathrm{Na}$ regressão linear múltipla, os estudantes que não leram material específico sobre suicídio $(\mathrm{p}<0,001)$, que tinham mais sentimentos negativos $(<0,001)$ e maior percepção da própria capacidade profissional $(p=0,001)$ tiveram escores reduzidos de direito ao suicídio, ou seja, tinham atitudes mais moralistas. E os que não participam de atividades religiosas $(\mathrm{p}<0,001)$ e tinham diagnóstico de transtorno mental $(\mathrm{p}=0,002)$, apresentam atitudes menos moralistas (Tabela 3$)$. 
Tabela 3 - Análise dos fatores associados às atitudes relacionadas ao direito ao suicídio, segundo modelo de regressão linear múltipla, 2018

\begin{tabular}{lcccc}
\hline \multicolumn{1}{c}{ Variável } & Parâmetro & p & \multicolumn{2}{c}{ IC 95\% } \\
\hline Participação atividades religiosas (não) & 4,078 & $<, 001$ & 3,019 & 5,137 \\
Leitura de material sobre suicídio (não) & $-2,128$ & $<, 001$ & $-3,170$ & $-1,086$ \\
Transtorno Mental (sim) & 2,058 &, $\mathbf{0 0 2}$ &, 779 & 3,337 \\
Período (8 ao 10 ${ }^{\circ}$ & $-1,078$ &, 050 & $-2,156$ &,- 001 \\
Esperança &,- 199 & $<, 001$ &,- 290 &,- 108 \\
F1- Sentimentos negativos &,- 192 & $<, 001$ &,- 291 &,- 093 \\
F2- Capacidade profissional autopercebida &,- 158 &, $\mathbf{0 0 1}$ &,- 254 &,- 062 \\
Constant & 24,808 & $<, 001$ & 21,165 & 28,451 \\
\hline
\end{tabular}

Modelo de regressão linear múltipla; p - valor de p; IC - intervalo de confiança. Fonte: Autores.

\section{Discussão}

Os escores médios obtidos no QUACS sobre o direito ao suicídio $(15,4)$ foram mais elevados do que os obtidos na competência profissional autopercebida $(11,3)$ e nos sentimentos negativos $(10,5)$, o que aponta maior concordância com afirmações menos moralistas do que com a competência profissional autopercebida ou com sentimentos negativos. Esses dados coadunam com estudos realizados com estudantes universitários, nos quais houve preponderância de atitude mais compreensiva e menos moralista dos entrevistados (Kirchner \& Queluz, 2019; Nunes et al., 2020).

O sexo feminino foi associado a mais sentimentos negativos e o sexo masculino foi associado a maior competência profissional e a atitudes menos moralistas (embora não tenha sido preditor desses desfechos). O direito ao suicídio não esteve associado ao sexo em estudos prévios (Moraes et al., 2016; Storino et al., 2018; Vedana \& Zanetti, 2019; Nunes et al., 2020). Um estudo realizado com estudantes de enfermagem também identificou mais sentimentos negativos entre as mulheres (Vedana \& Zanetti, 2019) e outros estudos encontraram maior percepção da capacidade profissional entre homens (Moraes et al., 2016; Storino et al., 2018; Nunes et al., 2020). Contudo, há investigações que não identificaram diferença estatisticamente significante entre o sexo e algumas das atitudes relacionadas ao comportamento suicida (Kirchner \& Queluz, 2019; Storino et al., 2018; Nunes et al., 2020).

No presente estudo, os estudantes heterossexuais apresentaram escores indicativos de mais sentimentos negativos e atitudes mais condenatórias relacionadas ao comportamento suicida. Tais resultados não puderam ser comparados, pois essa variável não foi avaliada em estudos prévios (Vedana \& Zanetti, 2019; Kirchner \& Queluz, 2019; Moraes et al., 2016; Storino et al., 2018; Nunes et al., 2020) sendo necessárias outras investigações sobre o assunto.

A participação em eventos sobre prevenção do suicídio teve associação com escores mais baixos de sentimentos negativos e mais elevados para capacidade profissional (embora essa variável não tenha sido preditora de tais desfechos). Investigações conduzidas com universitários brasileiros corroboram tais resultados (Botti et al., 2015; Kirchner \& Queluz, 2019; Picarelli, Hübner, \& Rodrigues, 2020; Nunes et al., 2020). O direito ao suicídio não esteve associado à participação em eventos no presente estudo e na literatura (Botega, 2015; Botti et al., 2015; Picarelli et al., 2020; Vedana \& Zanetti, 2019; Kirchner \& Queluz, 2019; Medeiros, Medeiros, \& Pinto, 2020; Nunes et al., 2020). É possível que atividades educativas tenham menor impacto na transformação do direito ao suicídio do que em outras atitudes (Moraes et al., 2016; Ramberg et al., 2016). 
A participação em cursos, capacitação e eventos é de grande relevância para promover mudanças de atitudes, menos sentimentos negativos e permitir o desenvolvimento de competências para uma assistência adequada ao indivíduo que apresenta comportamento suicida (Picarelli et al., 2020), ainda que se trate de treinamentos breves (Medeiros et al., 2020). Espera-se que cursos e treinamentos sobre prevenção de suicídio aumentem o conhecimento e reduzam as atitudes estigmatizantes (Öztürk \& Akin, 2018). É importante que as diferentes estratégias de capacitação (eventos, cursos e as disciplinas oferecidos durante e após a graduação) incorporem a abordagem de saberes atitudinais, cognitivos e procedimentais de maneira a potencializar as atitudes mais adequadas frente ao comportamento suicida (Moraes et al., 2016).

A leitura de materiais sobre suicídio esteve associada às atitudes relacionadas ao comportamento suicida, embora não tenha sido preditora desses desfechos. Os universitários que referiram ter lido material sobre o assunto apresentavam menos sentimentos negativos, maior competência profissional e atitudes menos moralistas. Esses dados corroboram parcialmente com investigações realizadas com graduandos de enfermagem brasileiros. Vedana e Zanetti (2019) identificaram que aqueles que leram material sobre o tema apresentavam menos sentimentos negativos e no estudo de Moraes, et al. (2016) os estudantes que relataram a leitura apresentavam atitudes menos condenatórias.

No presente estudo, o período do curso não foi uma variável associada a sentimentos negativos e competência profissional. A única associação encontrada foi de que estudantes do $5^{\circ}$ ao $7^{\circ}$ período apresentaram atitudes menos condenatórias, quando comparados aos estudantes do $8^{\circ}$ ao $10^{\circ}$ período. O período do curso apresenta resultados diversos em diferentes investigações. Estudo de Magalhães et al. (2014) não encontrou associação entre período do curso, sentimentos negativos e capacidade profissional e identificou que estudantes do último período tinham menos atitudes condenatórias. Em outra investigação, estudantes de medicina dos últimos períodos apresentavam menos sentimentos negativos, mais capacidade profissional, e maior percepção do direito ao suicídio (Nunes et al., 2020). Outra pesquisa revelou que etapas finais do curso estiveram associadas a maior presença de sentimentos negativos, menor capacidade profissional e menor percepção dos direitos ao suicídio (Kirchner \& Queluz, 2019). Esse fato pode ser justificado porque muitos profissionais se sentem confrontados em lidar com o comportamento suicida, uma vez que sua formação se fundamenta em salvar vidas (Braz, Ramos, \& Álvares, 2019).

É relevante mencionar que cursar disciplina sobre saúde mental ou psiquiatria não foi associado às atitudes relacionadas ao comportamento suicida. No estudo de Moraes et al. (2016) a participação em disciplina de Psiquiatria esteve associada a maior capacidade profissional. Os conteúdos e estratégias de ensino-aprendizagem podem ser importantes fatores envolvidos nesse processo. É importante incluir aspectos introdutórios da prevenção do comportamento suicida, como componente curricular para os cursos da área da saúde, considerando que essa demanda é frequente em diversos contextos clínicos. O déficit de conhecimento pode favorecer o despreparo dos profissionais para o atendimento, o que pode desencadear sentimentos e emoções negativas, prejudicando a abordagem a esses pacientes (Storino et al., 2018). Por outro lado, o maior conhecimento sobre o assunto pode reduzir comportamentos negativos, permitir reflexões e atitudes acolhedoras e uma assistência qualificada aos indivíduos com comportamento suicida (Botti et al., 2015; Nunes et al., 2020).

Não ter crença religiosa foi associado a atitudes menos condenatórias e foi fator preditor de maior percepção de competência profissional entre os universitários. Pessoas que negaram participar de atividades religiosas tiveram atitudes menos condenatórias. Pesquisa realizada com profissionais de saúde observou que ter religião e frequentar cultos religiosos não foram fatores associados às atitudes frente ao comportamento suicida (Storino et al., 2018). Estudo desenvolvido com estudantes enfermagem encontrou que a crença religiosa poderia estar associada a atitudes mais críticas sobre o comportamento suicida (Flood et al., 2018). Estudo conduzido com agentes comunitários de saúde observou que a filiação e a participação em atividades religiosas esteve associada a uma perspectiva mais moralista do suicídio (Medeiros et al., 2020). 
Neste estudo, observou-se a associação entre o direito ao suicídio (atitudes menos condenatórias) e insatisfação com o apoio social, diagnóstico de transtorno mental, tratamento com psicofármacos, acompanhamento em saúde mental, risco de suicídio, sintomas depressivos, baixa autoestima e desesperança. É possível que experiências ligadas ao sofrimento emocional possam favorecer as atitudes menos moralistas, compreensivas e empáticas na interação com pessoas com comportamento suicida. Por outro lado, tais sofrimentos podem propiciar o comportamento suicida e a defesa do direito ao suicídio como uma opção viável para si próprio. Estudo com estudantes universitários do curso de medicina da Áustria e da Turquia identificou que aqueles com maior comportamento suicida tinham mais aceitação em relação ao suicídio (Eskin et al., 2011). É importante que as atitudes relacionadas ao direito ao suicídio sejam cuidadosamente investigadas e abordadas em estratégias de formação e de psicoeducação, pois podem estar associadas a diferentes motivações, perspectivas e desfechos.

Menos sintomas depressivos, autoestima e esperança foram associados a maior competência profissional autopercebida, risco de suicídio e esperança foram preditores deste desfecho. Assim, alguns fatores indicativos de bem-estar podem estar associados a melhor percepção de competência para manejo do comportamento suicida. $\mathrm{O}$ atendimento às pessoas em comportamento suicida requer que o profissional esteja emocionalmente preparado (Reisdorfer et al., 2015; Braz et al., 2019). Profissionais de saúde com sintomas depressivos podem ter maior insegurança no desenvolvimento da assistência, principalmente mediante a possibilidade iminente de morte do paciente (Manetti \& Marziale, 2007). Além disso, o déficit na formação dos profissionais de saúde sobre a temática suicídio pode contribuir para o desconforto emocional (Oliveira et al., 2016). O apoio e o desenvolvimento de habilidades para a gestão de emoções associadas à dor, ao sofrimento e à morte precisam ser inseridos nos planos curriculares (Gomes \& Oliveira, 2013).

Foram identificadas correlações entre os três fatores que representam atitudes ligadas ao comportamento suicida. Os sentimentos negativos foram mais elevados quando a competência profissional e a compreensão do direito ao suicídio eram menores. Esse mesmo padrão foi observado em estudo realizado com estudantes de enfermagem portugueses (Vedana et al., 2020). A correlação negativa entre sentimentos negativos e a competência profissional foi documentada em estudo realizado com estudantes (Vedana \& Zanetti, 2019) e profissionais de enfermagem brasileiros (Vedana et al., 2017). Além disso, no presente estudo, a confiança profissional para lidar com os indivíduos com comportamento suicida foi maior entre pessoas com menor compreensão do direito ao suicídio, aspecto não identificado em estudos prévios (Vedana \& Zanetti, 2019; Vedana et al., 2020; Vedana et al., 2017).

Apresentou limitações que precisam ser consideradas, a primeira se refere ao delineamento transversal, o qual não permite estabelecer uma relação de causa e efeito entre as variáveis. Outra limitação do estudo foi ter sido realizado com graduandos a partir do $5^{\circ}$ período dos cursos da área da saúde, excluindo os universitários que se encontravam em períodos iniciais dos cursos, uma vez é fundamental investigar as atitudes relacionadas ao comportamento suicida do início ao final do curso, visto que essa população deve estar preparada para lidar com o indivíduo com comportamento suicida.

\section{Conclusão}

Entre as atitudes frente ao comportamento suicida avaliadas, foram encontradas menores médias de escores indicativos de sentimentos negativos relacionados a pessoas com comportamento suicida e maiores médias em escores relacionados à compreensão do direito ao suicídio.

Os preditores de sentimentos negativos relacionados a pessoas com comportamento suicida foram sexo feminino, não participar de atividades religiosas, menor competência profissional autopercebida e atitudes moralistas. Além dessas variáveis, estiveram associadas aos sentimentos negativos orientação sexual, apoio social, crença religiosa, prática de atividades religiosas, participar de eventos e leitura de material sobre suicídio. 
Foram preditores de competência profissional autopercebida, ter transtorno mental, ter mais esperança, presença risco de suicídio, não ter religião ou espiritualidade, menos sentimentos negativos e atitudes moralistas. Estiveram associadas aos sentimentos negativos, além dessas variáveis, sexo, participação de eventos e leitura de material sobre suicídio, sintomas depressivos e autoestima.

Os preditores de maior compreensão do direito ao suicídio foram não participam de atividades religiosas, ter diagnóstico de transtorno mental, ler material específico sobre suicídio, menos sentimentos negativos, menos esperança e menos percepção da própria capacidade profissional. Além dessas variáveis, estiveram associadas também sexo, orientação sexual, crença religiosa, satisfação com o apoio, período do curso, uso de psicofármacos, fazer acompanhamento em saúde mental, risco de suicídio, sintomas depressivos e autoestima.

Este estudo apresenta a contribuição de ter sido realizado com universitários de diversos cursos da área da saúde, o que se justifica uma vez que estes serão responsáveis por assistir o indivíduo com comportamento suicida. As contribuições elencadas no presente estudo podem subsidiar intervenções, investigações e programas de ensino para qualificar a formação de recursos humanos para a assistência à pessoa com comportamento suicida.

Sugere-se novas pesquisas com esta temática abordando universitários do início ao final da graduação, com o intuito de avaliar mudança de atitudes frente ao comportamento suicida durante esta trajetória. É válida a condução de novos delineamentos de estudo, com esta mesma temática e população.

\section{Referências}

Altmann, T. (2008). Attitude: a concept analysis. Nursing Forum. 43(3), 144-150. 10.1111/j.1744-6198.2008.00106.x

Amorim, P. (2000). International Neuropsychiatric Interview (MINI): validação de entrevista breve para diagnóstico de transtornos mentais. Brazilian Journal of Psychiatry, 22(3), 106-115. https://doi.org/10.1590/S1516-44462000000300003

Aragão, J. Introdução aos estudos quantitativos utilizados em pesquisas científicas (2011). Revista Práxis, 3(6), 59-62. https://doi.org/10.25119/praxis-3-6-566.

Botega, J. N. (2015). Crise Suicida: avaliação e manejo. Artmed.

Botega, N. J., Reginato, D. G., Silva, S. V. da, Cais, C. F. da S., Rapeli, C. B., Mauro, M. L. F., Cecconi, J. P., \& Stefanello, S. (2005). Nursing personnel attitudes towards suicide: the development of a measure scale. Brazilian Journal of Psychiatry, 27(4), 315-318. https://dx.doi.org/10.1590/S151644462005000400011

Botti, N. C. L., Araújo, L. M. C. de, Costa, E. E., \& Almeida, J. S. de (2015). Nursing students attitudes across the suicidal behavior. Invest Educ Enferm, 33(2), 334-342. 10.17533/udea.iee.v33n2a16

Braz, T. C. O., Ramos, T. de J. C. A., \& Álvares, A. da C. M. (2019). Intervenção de enfermagem no âmbito de tentativas de pacientes autoextermínios em emergência hospitalar. Revista de Iniciação Científica e Extensão,2(4), 241-246. https://revistasfacesa.senaaires.com.br/index.php/iniciacaocientifica/article/view/264

Carmona-Navarro, M. C., \& Pichardo-Martinez, M. C. (2012). Atitudes do profissional de enfermagem em relação ao comportamento suicida: influência da inteligência emocional. Revista Latino-Americana de Enfermagem, 20(6), 8 telas. Recuperado de https://www.scielo.br/pdf/rlae/v20n6/pt_19.pdf

Emergency Nurses Association (ENA). (2012). Clinical Practice Guideline: Suicide Risk Assessment Full Version.

Eskin, M., Voracek, M., Stieger, S., \& Altinyazar, V. (2011). A cross-cultural investigation of suicidal behavior and attitudes in Austrian and Turkish medical students. Social psychiatry and psychiatric epidemiology, 46(9), 813-823. https://doi.org/10.1007/s00127-010-0254-7

Flood, C., Yilmaz, M., Phillips, L., Lindsay, T., Eskin, M., Hiley, J., \& Tasdelen, B. (2018). Nursing students' attitudes to suicide and suicidal persons: A cross-national and cultural comparison between Turkey and the United Kingdom. Journal of psychiatric and mental health nursing, 25(7), 369-379. https://doi.org/10.1111/jpm.12461

Giacchero Vedana, K. G., Magrini, D. F., Zanetti, A., Miasso, A. I., Borges, T. L., \& Dos Santos, M. A. (2017). Attitudes towards suicidal behaviour and associated factors among nursing professionals: A quantitative study. Journal of psychiatric and mental health nursing, 24(9-10), 651-659. https://doi.org/10.1111/jpm.12413

Gomes, N. S., \& Silva, S. R. (2013). Avaliação da autoestima de mulheres submetidas à cirurgia oncológica mamária. Texto \& contexto enfermagem, 22(2), 509-516. http://dx.doi.org/10.1590/S0104-07072013000200029

Gomes, R. K., \& Oliveira, V. B. de. (2013). Depressão, ansiedade e suporte social em profissionais de enfermagem. Boletim de Psicologia, 63(138), 23-33. Recuperado de http://pepsic.bvsalud.org/scielo.php?script=sci_arttext\&pid=S0006-59432013000100004\&lng=pt\&tlng=pt 
Hutz, C. S., \& Zanon, C. (2011). Revisão da adaptação, validação e normatização da Escala de Autoestima de Rosenberg. Avaliação Psicológica, 10(1), p. 4149. http://pepsic.bvsalud.org/scielo.php?script=sci_arttext\&pid=S1677-04712011000100005\&lng=pt\&tlng=pt

Kelly, M., McCarthy, S., \& Sahm, L. J. (2014) Knowledge, attitudes and beliefs of patients and carers regarding medication adherence: a review of qualitative literature. European Journal of Clinical Pharmacology, 70(12), 1423-1431. https://doi.org/10.1007/s00228-014-1761-3

Kim, S. M., Baek, J. H., Han, D. H., Lee, Y. S., \& Yurgelun-Todd, D. A. (2015). Psychosocial -Environmental risk factors for suicide attempts in adolescents with suicidal ideation: findings from a sample of 73,238 adolescents. Suicide and Life-Threatening Behavior, 45(4), 477-487. https://doi.org/10.1111/sltb.12143

Kirchner, L. F., \& Queluz, F. N. F. R. (2019). Conhecimento e atitudes de universitários acerca do suicídio: influências sociodemográficas e acadêmicas. Brazilian Journal of Health Review, 2(4), 3120-3130. https://doi.org/10.34119/bjhrv2n4-077

Magalhães, C. A., Neves, D. M. M., Brito, L. M. D. M., Leite, B. B. C., Pimenta, M. M. de F., \& Vidal, C. E. L. (2014). Atitudes de estudantes de medicina em relação ao suicídio. Revista Brasileira de Educação Médica, 38 (4), 470-476. https://dx.doi.org/10.1590/S0100-55022014000400008

Manetti, M. L., \& Marziale, M. H. P. (2007). Fatores associados à depressão relacionada ao trabalho de enfermagem. Estudos de Psicologia (Natal), 12(1), 7985. DOI: $10.1590 / \mathrm{S} 1413-294 \mathrm{X} 2007000100010$

Medeiros, B. G. de, Medeiros, N. de S. B., \& Pinto, T. R. (2020). Educação permanente em saúde mental: o suicídio na agenda do cuidado dos Agentes Comunitários de Saúde. Pesquisas e práticas psicossociais, 15(2), 1-16. http://pepsic.bvsalud.org/scielo.php?script=sci_arttext\&pid=S1809$89082020000200011 \& \operatorname{lng}=\mathrm{pt} \& \operatorname{lng}=\mathrm{pt}$

Menon, V. (2013). Suicide risk asssessment and formulation: an update. Asian Journal of Psychiatry, 6(5), 430-435. https://doi.org/10.1016/j.ajp.2013.07.005

Miot, H. A. (2018). Análise de correlação em estudos clínicos e experimentais. Jornal Vascular Brasileiro, 17(4), 275-279. https://doi.org/10.1590/1677-5449.174118

Moraes, S. M., Magrini, D. F., Zanetti, A. C. G., Santos, M. A. dos, \& Vedana, K. G. G. (2016). Atitudes relacionadas ao suicídio entre graduandos de enfermagem e fatores associados. Acta Paulista de Enfermagem, 29(6), 643-649. https://doi.org/10.1590/1982-0194201600090

Nebhinani, M., Nebhinani, N., Tamphasana, L., \& Gaikwad, A. D. (2013). Nursing students attitude towards suicide attempters: A study from rural part of Northern India. Journal of neurciences in rural practice, 4 (4), 400-407. 10.4103 / 0976-3147.120240

Nunes, E. A., Oliveira, B. B., Galvão, L. B. L. (2020). Atitudes de estudantes de medicina diante do comportamento suicida e fatores associados. Revista Brasileira de Educação Médica, 44(4), e131. https://doi.org/10.1590/1981-5271v44.4-20200021

Oliveira, C. T. de, Collares, L. A., Noal, M. H. O., \& Dias, A. C. G. (2016). Percepções de uma equipe de saúde mental sobre o comportamento suicida. Gerais: $\quad$ Revista Interinstitucional de Psicologia,9(1), 78-89. http://pepsic.bvsalud.org/scielo.php?script=sci_arttext\&pid=S1983$82202016000100007 \& \operatorname{lng}=\mathrm{pt} \& \operatorname{lng}=\mathrm{pt}$

Öztürk, A., \& Akin, S. (2018). Evaluation of knowledge level about suicide and stigmatizing attitudes in university students toward people who commit suicide. Journal of Psychiatric Nursing, 9(2), 96-104, 2018. https://doi.org/10.14744/phd.2018.49389

Parcias, S., Rosario, B. P. do, Sakae, T., Monte, F., Guimarães, A. C. A., \& Xavier, A. J. (2011). Validação da versão em português do Inventário de Depressão Maior. Jornal Brasileiro de Psiquiatria, 60(3), 164-170. https://doi.org/10.1590/S0047-20852011000300003

Pereira, A. S., Willhelm, A. R., Koller, S. H., \& Almeida, R. M. M. de. (2018). Fatores de risco e proteção para tentativa de suicídio na adultez emergente. Ciência \& Saúde Coletiva, 23(11), 3767-3777. https://doi.org/10.1590/1413-812320182311.29112016

Picarelli, C., Hübner, C., \& Rodrigues, C. (2020). Prevenção de suicídio: modificando percepção e conhecimento de estudantes de medicina. Psicologia, Saúde \& Doenças, 21(2), 446-455. https://dx.doi.org/10.15309/20psd210219

Polit, D. F., \& Beck, C. T. (2011). Fundamentos de pesquisa em enfermagem. Artmed.

Ramberg, I. L., Di Lucca, M. A., \& Hadlaczky, G. (2016). The Impact of Knowledge of Suicide Prevention and Work Experience among Clinical Staff on Attitudes towards Working with Suicidal Patients and Suicide Prevention. International journal of environmental research and public health, 13(2), 195. https://doi.org/10.3390/ijerph13020195

Reisdorfer, N., Araújo, G. M. de, Hildebrandt, L. M., Gewehr, T. R., Nardino, J., \& Leite, M. T. (2015). Suicídio na voz de profissionais de enfermagem e estratégias de intervenção diante do comportamento suicida. Revista de Enfermagem da UFSM, 5(2), 295-304. https://doi.org/10.5902/2179769216790

Sartore, A. C., \& Grossi, S. A. A. (2008). Escala de Esperança de Herth: instrumento adaptado e validado para a língua portuguesa. Revista da Escola de Enfermagem da USP, 42(2), 227-232. https://doi.org/10.1590/S0080-62342008000200003

Saunders, K. E, Hawton, K., Fortune, S., Farrell, S. (2012). Attitudes and knowledge of clinical staff regarding people who self-harm: A systematic review. Journal of Affective Disorders, 139(3), p. 205-216. https://doi.org/10.1016/j.jad.2011.08.024

Schlösser, A., Rosa, G. F. C., \& More, C. L. O. O. (2014). Revisão: comportamento suicida ao longo do ciclo vital. Temas em Psicologia, 22(1), 133-145. https://dx.doi.org/10.9788/TP2014.1-11

Siqueira, A. L., Tibúrcio, J. D. Estatística na área da saúde: conceitos, metodologia, aplicações e prática computacional. (2011). Coopmed.

Storino, B. D., Campos, C. F. e, Chicata, L. C. de O., Campos, M. de A., Matos, M. S. da C., Nunes, R. M. C. M., \& Vidal, C. E. L. (2018). Atitudes de profissionais da saúde em relação ao comportamento suicida. Cadernos Saúde Coletiva, 26(4), 369-377. https://doi.org/10.1590/1414-462x201800040191

Talseth, A. G., Gilje, F. Unburdening suffering: responses of psychiatrists to patients' suicide deaths. Nursing Ethics, 14(5), 620-636, https://doi.org/10.1177/0969733007080207 
Research, Society and Development, v. 10, n. 5, e25210514804, 2021

(CC BY 4.0) | ISSN 2525-3409 | DOI: http://dx.doi.org/10.33448/rsd-v10i5.14804

Vedana, K. G. G., \& Zanetti, A. C. G. (2019). Attitudes of nursing students toward to the suicidal behavior. Revista. Latino-Americana Enfermagem, 27, e3116. 10.1590 / 1518-8345.2842.3116

Vedana, K. G. G., Santos, J. C., Zanetti, A. C. G, Miasso, A. I. (2020). Attitudes of Portuguese nursing undergraduates students toward suicidal behaviour. British Journal of Mental Health Nursing. 9(1), 41-48. https://doi.org/10.12968/bjmh.2018.0031

Werlang, B. S. G., Borges, V. R., \& Fensterseifer, L. (2005). Indícios de potencial suicida na adolescência. Psicologia Revista, 14(1), 41-57. https://revistas.pucsp.br/index.php/psicorevista/article/view/18125/13480

World Health Organization (WHO). (2000). Prevenção do suicídio: Um manual para profissionais da saúde em atenção primária. Transtornos mentais e comportamentais. Departamento de Saúde Mental.

Zadravec, T. \& Grad, O. (2013). Origins of suicidality: compatibility of layand expert belirfs-Qualitative study. Pshychiatria Danubina, 25(2), 152-155. https://pubmed.ncbi.nlm.nih.gov/23793279/ 